\title{
Anti-biofilm activity and synergism of novel thiazole compounds with glycopeptide antibiotics against multidrug-resistant Staphylococci
}

\author{
Haroon Mohammad ${ }^{1}$, Abdelrahman S Mayhoub ${ }^{2}$, Mark Cushman ${ }^{3}$ and Mohamed N Seleem ${ }^{1}$ \\ Methicillin-resistant Staphylococcus aureus (MRSA) infections are a leading cause of death among all fatalities caused by \\ antibiotic-resistant bacteria. With the rise of increasing resistance to current antibiotics, new antimicrobials and treatment \\ strategies are urgently needed. Thiazole compounds have been shown to possess potent antimicrobial activity. A lead thiazole 1 \\ and a potent derivative 2 were synthesized and their activity in combination with glycopeptide antibiotics was determined against \\ an array of MRSA and vancomycin-resistant $S$. aureus (VRSA) clinical isolates. In addition, the anti-biofilm activity of the novel \\ thiazoles was investigated against $S$. epidermidis. Compound 2 behaved synergistically with vancomycin against MRSA and was \\ able to resensitize VRSA to vancomycin, reducing its MIC by 512 -fold in two strains. In addition, both thiazole compounds were \\ superior to vancomycin in significantly reducing $S$. epidermidis biofilm mass. Collectively, the results obtained demonstrate that \\ compounds 1 and 2 possess potent antimicrobial activity alone or in combination with vancomycin against multidrug-resistant \\ staphylococci and show potential for use in disrupting staphylococcal biofilm.
}

The Journal of Antibiotics (2015) 68, 259-266; doi:10.1038/ja.2014.142; published online 15 October 2014

\section{INTRODUCTION}

Antibiotic-resistant bacteria are a major global health concern resulting in 23000 deaths each year in the United States alone. ${ }^{1}$ Two species alone, methicillin-resistant Staphylococcus aureus (MRSA) and S. epidermidis, are responsible annually for the majority of skin and soft-tissue infections, and infections caused by bacterial biofilms present on indwelling medical devices. ${ }^{2,3}$ Biofilms are responsible for $80 \%$ of microbial infections that develop in the human body and bacterial biofilms on implanted biomedical devices and tissue surfaces (chronic wound) constitute an ever-increasing threat to human health and place a significant burden on healthcare systems. ${ }^{4}$ Biofilms consist of a cluster of bacterial cells enclosed within an extracellular matrix that collectively attach to an animate or inanimate surface. ${ }^{4}$ The cells present within a biofilm pose a key challenge as they demonstrate increased resistance to the effect of antimicrobials. ${ }^{5}$

Antibiotics have been key allies in the treatment of bacterial infections for more than 80 years. Although several classes of antibiotics were once capable of treating staphylococci-induced infections, strains have emerged that are resistant to an array of antimicrobials once deemed effective including $\beta$-lactams, ${ }^{6}$ macrolides $^{2}$ and fluoroquinolones. ${ }^{6,7}$ Further exacerbating the issue is the rise of strains (such as vancomycin-resistant S. aureus (VRSA)), which are resistant to antibiotics deemed drugs of last resort for treatment of staphylococcal infections, including glycopeptide antibiotics like vancomycin. ${ }^{8}$ Conventional antibiotics face an added challenge in the treatment of biofilm infections, as bacteria present within a biofilm may be 1000 -fold more resistant to antibiotics compared with their planktonic equivalents. ${ }^{5}$ Thus, there is a critical need for the discovery of novel antimicrobials and treatment strategies to circumvent this growing public health concern.

Several thiazole compounds have been shown to be effective anticonvulsant, ${ }^{9}$ anticancer ${ }^{10,11}$ and antiviral agents. ${ }^{12}$ However, limited studies have been performed to characterize their abilities as antimicrobial agents, particularly against MRSA. Darwish et al., ${ }^{13}$ synthesized a series of thiadiazole analogs incorporating a sulfonamide group and found that they possessed activity against Streptococcus pneumoniae and Bacillus subtilis. In addition, Desai et al. ${ }^{14}$ constructed a series of novel hybrid compounds that combined the thiazole and 1,3,4-oxadiazole pharmacophores, but found that they had limited activity against $S$. aureus (MIC of 6 of the 12 analogs constructed was $500 \mu \mathrm{g} \mathrm{ml}^{-1}$ or higher). Furthermore, a third study assessing a series of disubstituted 1,3-thiazole derivatives found the most potent analog possessed modest activity against a single strain of $S$. aureus tested (MIC of $50 \mu \mathrm{g} \mathrm{ml}^{-1}$ ). ${ }^{15}$ None of these studies assessed broader therapeutic applications of thiazole compounds beyond the use as single agent to inhibit bacterial growth in vitro.

\footnotetext{
${ }^{1}$ Department of Comparative Pathobiology, Purdue University College of Veterinary Medicine, West Lafayette, IN, USA; ${ }^{2}$ Department of Organic Chemistry, Al Azhar University, Cairo, Egypt and ${ }^{3}$ Department of Medicinal Chemistry and Molecular Pharmacology, Purdue University College of Pharmacy and the Purdue Center for Cancer Research, West Lafayette, IN, USA

Correspondence: Professor MN Seleem, Department of Comparative Pathobiology, Purdue University College of Veterinary Medicine, 625 Harrison Street, West Lafayette, IN 47907, USA. 
We recently discovered a novel lead thiazole compound 1 that exhibited potent antimicrobial activity against MRSA (Figure 1). ${ }^{16}$ The lead compound is composed of a thiazole nucleus connected to a cationic amino head at the C5-position and a lipophilic phenylalkyl tail at the C2-position. The aims of the present study are to identify whether the lead compound $\mathbf{1}$ and the most potent synthesized derivative $\mathbf{2}$ have potential to be used in combination with glycopeptide antibiotics, commonly used to treat MRSA infections, to analyze the ability of $\mathbf{1}$ and $\mathbf{2}$ to resensitize VRSA strains to glycopeptide antibiotics, and to assess whether these compounds are capable of disrupting staphylococcal biofilms using an in vitro model of S. epidermidis. Results garnered from this study will provide valuable insight into potential therapeutic applications of thiazole compounds for use as antibacterial agents.

\section{MATERIALS AND METHODS}

\section{Bacterial strains and reagents}

The bacterial strains of methicillin-resistant and vancomycin-intermediate S. aureus utilized were obtained from the Network on Antimicrobial Resistance in S. aureus. S. epidermidis ATCC 35984 was obtained from the American Tissue Culture Collection. Antibiotics were purchased commercially from Gold Biotechnology (St Louis, MO, USA) (vancomycin hydrochloride) and Biotang (Waltham, MA, USA) (teicoplanin). Both antibiotics were dissolved in dimethyl sulfoxide to obtain a stock $10 \mathrm{~mm}$ solution.

\section{Synthesis of thiazole compounds 1 and 2}

The detailed synthetic protocols and spectral data of final products $\mathbf{1}$ and $\mathbf{2}$, as well as all intermediates have been previously reported. ${ }^{16,17}$

\section{Determination of MIC and MBC against MRSA, VISA, VRSA and S. epidermidis}

The MICs of the thiazole compounds, vancomycin and teicoplanin against MRSA, vancomycin-intermediate S. aureus (VISA), VRSA and S. epidermidis were determined using the broth microdilution method, in accordance with the recommendations contained in the CLSI guidelines (with the exception that Mueller-Hinton broth (MHB) was used instead of cation-adjusted MHB). ${ }^{18}$ Bacteria were prepared in phosphate-buffered saline (PBS) until a McFarland standard of 0.5 was achieved. The solution was diluted 1:300 in MHB to reach a starting inoculum of $1 \times 10^{5} \mathrm{CFU} \mathrm{ml}^{-1}$. Bacteria were transferred to a 96-well microtiter plate. Thiazole compounds and antibiotics were added (in triplicate) to wells in the first row of the microtitier plate and then serially diluted along the ordinate. The plate was incubated at $37^{\circ} \mathrm{C}$ for $20-24 \mathrm{~h}$ before the MIC was determined. The MIC was categorized as the concentration at which no visible growth of bacteria was observed in a particular well.

The MBC was determined by plating $5 \mu \mathrm{l}$ from wells on the 96-well microtiter plate (where the MIC was determined) were no growth was observed onto tryptic soy agar (TSA) plates. The TSA plates were then incubated at $37^{\circ} \mathrm{C}$ for $20-24 \mathrm{~h}$ before the MBC was determined. The MBC was categorized as the concentration where $\geqslant 99 \%$ reduction in bacterial cell count was observed.

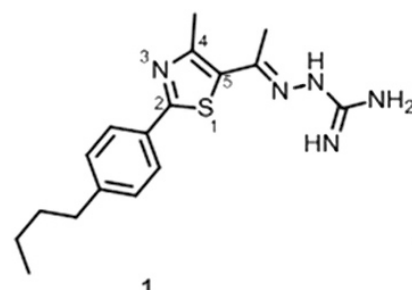

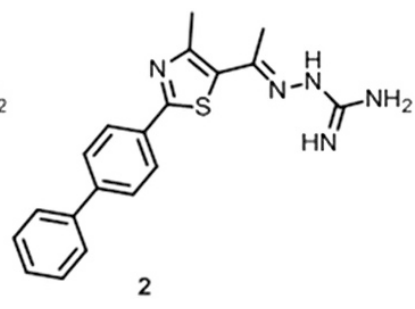

Figure 1 Chemical structure of thiazole compounds 1 and $\mathbf{2}$.
Time-kill analysis of thiazole compounds and glycopeptide antibiotics against MRSA

MRSA NRS123 (USA400) cells in the logarithmic growth phase were diluted to $\sim 1 \times 10^{8} \mathrm{CFU} \mathrm{ml}^{-1}$ and exposed to concentrations equivalent to 2,4 and $8 \times$ MIC (in triplicate) of thiazole compounds 1 and 2 , teicoplanin and vancomycin in MHB. Samples $(20 \mu \mathrm{l})$ were collected after $0,2,4,6,8,10$, 12 and $24 \mathrm{~h}$ of incubation at $37^{\circ} \mathrm{C}$ and subsequently serially diluted in PBS. Bacteria were then transferred to TSA plates and incubated at $37^{\circ} \mathrm{C}$ for $18-20 \mathrm{~h}$ before viable $\mathrm{CFU} \mathrm{ml}^{-1}$ was determined. The test agent was deemed bactericidal whether it successfully produced a $3-\log _{10}$ reduction in the bacterial count within $24 \mathrm{~h}$, as reported elsewhere. ${ }^{19}$

\section{Single-step resistance selection}

The frequency of spontaneous single-step resistance of the thiazole compounds and glycopeptide antibiotics to five MRSA strains was determined as reported elsewhere. ${ }^{20,21}$ Briefly, bacterial cultures $\left(>1 \times 10^{9} \mathrm{CFU} \mathrm{ml}^{-1}\right)$ were spread onto Mueller-Hinton agar plates (10-mm diameter) containing each compound/antibiotic at $4 \times$ MIC. Plates were incubated aerobically at $37^{\circ} \mathrm{C}$ for $48 \mathrm{~h}$. The frequency of resistance was calculated as the number of resistant colonies per inoculum. ${ }^{21}$

\section{Combination therapy analysis of thiazole compounds with glycopeptide antibiotics}

The relationship between the thiazole compounds and glycopeptide antibiotics (vancomycin and teicoplanin) was assessed via a standard checkerboard assay. ${ }^{22}$ Bacteria equivalent to a McFarland standard of 0.5 were prepared in PBS. The bacteria were then diluted in MHB to achieve a starting cell density of $1 \times 10^{5}$ $\mathrm{CFU} \mathrm{m}{ }^{-1}$. The MHB was transferred to all wells of a 96-well microtiter plate. The thiazole compounds and glycopeptide antibiotics were diluted in MHB to achieve a starting concentration equivalent to $2 \times$ or $4 \times \mathrm{MIC}$, respectively. The glycopeptide antibiotic was serially diluted along the abscissa of the microtiter plate, whereas the thiazole compound was serially diluted along the ordinate. The plate was incubated for $20-24 \mathrm{~h}$ at $37^{\circ} \mathrm{C}$. The MIC of the test compound, in combination with each glycopeptide antibiotic studied, was determined as the lowest concentration of each compound/antibiotic where no visible growth of bacteria was observed. The fractional inhibitory concentration index $(\Sigma \mathrm{FIC})$ was calculated for each combination as follows:

$$
\sum \mathrm{FIC}=
$$

$\left(\frac{\text { MIC thiazole compound in combination with glycopeptide antibiotic }}{\text { MIC thiazole compond alone }}\right)$ $+\left(\frac{\text { MICglycopeptide antibiotic in combination with thiazole compound }}{\text { MIC glycopeptide antibiotic }}\right)$

A synergistic relationship was classified as an FIC index $\leqslant 0.5$. FIC values above 0.5 but $<4.0$ were characterized as indifference, whereas FIC values above 4.0 were classified as antagonistic.

\section{Resensitization of VRSA strains to vancomycin using broth microdilution method}

The MHB was inoculated with VRSA $\left(5 \times 10^{5} \mathrm{CFU} \mathrm{ml}{ }^{-1}\right)$, as described elsewhere. ${ }^{23}$ Aliquots $(5 \mathrm{ml})$ of the bacterial suspension were divided into microcentrifuge tubes. Compound 1 or 2 (at $1 / 2 \times \mathrm{MIC}$ ) was introduced into each tube. After sitting at room temperature for $30 \mathrm{~min}, 1 \mathrm{ml}$ of sample from each tube was transferred to a new centrifuge tube prior to addition of the antibiotic (either vancomycin or teicoplanin at a concentration equivalent to their MIC). Using a 96-well microtiter plate, rows 2-12 were filled with the remaining $4 \mathrm{ml}$ bacterial suspension (containing either compound 1 or 2). Aliquots $(200 \mu \mathrm{l})$ from tubes containing both the thiazole compound and glycopeptide antibiotic were transferred to row 1 of the 96-well plate. After aspirating contents in the first row for 4-6 times, $100 \mu \mathrm{l}$ was transferred from wells in row 1 to row 2 . This process was repeated to dilute the remaining wells containing no antibiotic. Untreated bacteria served as a control. The plate was incubated at $37^{\circ} \mathrm{C}$ for $20 \mathrm{~h}$ before the MIC was recorded. The MIC was categorized as the concentration at which no visible growth of bacteria was 
observed in a particular well. A fold reduction was calculated by comparing the MIC of the antibiotic alone with the MIC of the antibiotic given in combination with 1 or 2 .

\section{Staphylococcus biofilm mass reduction determination}

The microtiter dish biofilm formation assay ${ }^{24}$ was used to assess the ability of the thiazole compounds to disrupt an adherent staphylococcal biofilm, similar to what has been described elsewhere. ${ }^{25}$ S. epidermidis ATCC 35984 was transferred to tryptic soy broth and incubated at $37^{\circ} \mathrm{C}$ for $24 \mathrm{~h}$ before being diluted 1:200 in tryptic soy broth $+1 \%$ glucose. This solution was transferred to each well of a 96 -well microtiter plate and incubated at $37^{\circ} \mathrm{C}$ for $24 \mathrm{~h}$ to permit biofilm formation on the well surface. Bacteria were removed and wells were washed twice with PBS. Compounds 1, 2, or vancomycin were added (in triplicate) to wells and serially diluted. The microtiter plate was then incubated at $37^{\circ} \mathrm{C}$ for $24 \mathrm{~h}$. The plate was washed twice by submerging in deionized water. Crystal violet $(0.1 \%(\mathrm{w} / \mathrm{v}))$ was added to each well and allowed to stain the biofilm for $20 \mathrm{~min}$ before addition of $95 \%$ ethanol to decolorize. Using a kinetic microplate reader (Molecular Devices, Sunnyvale, CA, USA), the OD of each well at $595 \mathrm{~nm}$ was measured. Percent biofilm mass reduction was calculated for each treatment regimen as compared with the control (wells receiving no treatment).

\section{Kinetic solubility determination of compound 2}

Serial dilutions of compound 2 were prepared in DMSO at $100 \times$ the final concentration. Compound 2 was then diluted 100 -fold into PBS in a 96-well plate and mixed. The absorbance of the PBS-containing plate was measured prior to addition of the test agents to determine the background absorbance. After $2 \mathrm{~h}$, the presence of precipitate was detected by turbidity (absorbance at $540 \mathrm{~nm})$. An absorbance value of greater than (mean $+3 \times$ s.d. of the blank), after subtracting the pre-experiment background, was indicative of turbidity. The solubility limit is reported as the highest experimental concentration for compound 2 with no evidence of turbidity.

\section{Caco-2 bidirectional permeability assessment of compound 2}

To assess the ability of compound $\mathbf{2}$ to passively permeate through epithelial tissue, a Caco-2 permeability assay was performed as described previously. ${ }^{16}$ Caco-2 cells grown in tissue culture flasks were trypsinized, suspended in medium, and the suspensions were applied to wells of a Millipore 96-well Caco-2 plate (Millipore, Billerica, MA, USA). The cells were allowed to grow and differentiate for 3 weeks, feeding at 2-day intervals. For apical to basolateral $(\mathrm{A} \rightarrow \mathrm{B})$ permeability, compound $\mathbf{2}$ was added to the apical $(\mathrm{A})$ side and amount of permeation was determined on the basolateral (B) side; for basolateral to apical $(\mathrm{B} \rightarrow \mathrm{A})$ permeability, compound 2 was added to the $\mathrm{B}$ side and the amount of permeation was determined on the A side. The A-side buffer contained $100 \mu \mathrm{M}$ lucifer yellow dye, in transport buffer $\left(1.98 \mathrm{gl}^{-1}\right.$ glucose in $10 \mathrm{~mm}$ HEPES, $1.0 \times$ Hank's balanced salt solution) at $\mathrm{pH} 6.5$, and the B-side buffer contained transport buffer at $\mathrm{pH}$ 7.4. Caco-2 cells were incubated with these buffers for $2 \mathrm{~h}$, and the receiver side buffer was removed for analysis by $\mathrm{LC} /$ MS/MS. To verify the Caco-2 cell monolayers were properly formed, aliquots of the cell buffers were analyzed by fluorescence to determine the transport of the impermeable dye lucifer yellow. Any deviations from control values are reported. Data are expressed as permeability $P$ app $=\frac{-\left(\frac{\mathrm{d} d}{\mathrm{dt}}\right)}{C_{0} A}$ where $\frac{\mathrm{dQ}}{\mathrm{dt}}$ is the rate of permeation, $C_{0}$ is the initial concentration of test agent, and $A$ is the area of the monolayer. In bidirectional permeability studies, the efflux ratio $\left(R_{\mathrm{E}}\right)$ is also calculated: $R_{\mathrm{E}}=\frac{P \operatorname{app}(\mathrm{B} \rightarrow \mathrm{A})}{\operatorname{Papp}(\mathrm{A} \rightarrow \mathrm{B})}$. An $R_{\mathrm{E}}>2$ indicates a potential substrate for P-glycoprotein or other active efflux transporters.

\section{Statistical analysis}

All statistical analysis was performed using the two-tailed Student's $t$-test $(P<0.05)$ using Microsoft Excel software (Redmond, WA, USA).

\section{RESULTS AND DISCUSSION}

Determination of the antimicrobial activity of the thiazole compounds and glycopeptide antibiotics

We have designed and synthesized a series of thiazole derivatives containing modifications to the lipophilic alkyl side chain of $1 .{ }^{16}$ The

Table 1 MIC and MBC of thiazole compounds 1 and 2, teicoplanin and vancomycin against MRSA, VISA and VRSA strains

\begin{tabular}{|c|c|c|c|c|c|c|c|c|}
\hline \multirow[b]{3}{*}{ Strain } & \multicolumn{8}{|c|}{ MIC and MBC ( $\left.\mu g \mathrm{ml}^{-1}\right)$ of thiazole compounds, teicoplanin and vancomycin against S. aureus } \\
\hline & \multicolumn{2}{|c|}{1} & \multicolumn{2}{|c|}{2} & \multicolumn{2}{|c|}{ Teicoplanin } & \multicolumn{2}{|c|}{ Vancomycin } \\
\hline & MIC & $M B C$ & $M I C$ & $M B C$ & $M I C$ & $M B C$ & $M I C$ & $M B C$ \\
\hline \multicolumn{9}{|l|}{ MRSA } \\
\hline NRS107 & 1.38 & 2.77 & 1.40 & 1.40 & 0.94 & 0.94 & 0.74 & 1.49 \\
\hline NRS119 & 1.38 & 1.38 & 1.40 & 1.40 & 0.94 & 0.94 & 0.74 & 1.49 \\
\hline NRS123 (USA400) & 1.38 & 11.07 & 1.40 & 5.58 & 0.94 & 15.04 & 0.37 & 0.37 \\
\hline NRS194 & 1.38 & 5.54 & 1.40 & 11.17 & 0.94 & 15.04 & 0.74 & 0.74 \\
\hline NRS384 (USA300) & 1.38 & 1.38 & 1.40 & 2.80 & 0.94 & 15.04 & 0.74 & 0.74 \\
\hline NRS385 (USA500) & 1.38 & 1.38 & 1.40 & 1.40 & 0.94 & 3.76 & 0.74 & 0.74 \\
\hline ATCC 43300 & 1.38 & 2.77 & 1.40 & 2.80 & 0.94 & 3.76 & 0.74 & 0.74 \\
\hline \multicolumn{9}{|l|}{ VISA } \\
\hline NRS1 & 1.38 & 1.38 & 0.70 & 1.40 & 3.76 & 7.52 & 2.97 & 2.97 \\
\hline NRS19 & 1.38 & 1.38 & 1.40 & 1.40 & 0.94 & 0.94 & 2.97 & 2.97 \\
\hline NRS37 & 1.38 & 1.38 & 0.70 & 1.40 & 7.52 & 7.52 & 2.97 & 2.97 \\
\hline \multicolumn{9}{|l|}{ VRSA } \\
\hline VRS1 & 2.77 & 2.77 & 1.40 & 1.40 & 120.30 & $>240.60$ & 760.68 & 760.68 \\
\hline VRS4 & 2.77 & 2.77 & 0.70 & 2.80 & 60.15 & 60.15 & 760.68 & 760.68 \\
\hline VRS5 & 2.77 & 5.54 & 1.40 & 5.58 & 60.15 & 60.15 & 760.68 & 760.68 \\
\hline \multicolumn{9}{|l|}{ S. epidermidis } \\
\hline ATCC 35984 & 2.77 & ND & 0.70 & ND & ND & ND & 0.74 & ND \\
\hline
\end{tabular}

Abbreviations: MRSA, methicillin-resistant S. aureus; ND, not determined; VISA, vancomycin-intermediate $S$. aureus; VRSA, vancomycin-resistant S. aureus. 
antimicrobial susceptibility analysis of these derivatives, using the standard broth microdilution assay, ${ }^{26}$ revealed compound 2 exhibited the most potent antibacterial activity against multidrug-resistant staphylococci. As Table 1 demonstrates, the MIC for compound 1 was $1.38 \mu \mathrm{g} \mathrm{ml}^{-1}$; compound 2 showed similar activity inhibiting growth of the same strains at a concentration of $1.40 \mu \mathrm{g} \mathrm{ml}^{-1}$. At these concentrations, the compounds are not toxic to mammalian cells, as confirmed in a previous study. ${ }^{16}$

The thiazole compounds exhibited activity against MRSA strains resistant to several different classes of antibiotics, including macrolides (NRS384), fluoroquinolones (NRS385), aminoglycosides (NRS385), tetracyclines (NRS384) and oxazolidinones (NRS119). In addition, both 1 (MIC from 1.38 to $2.77 \mu \mathrm{g} \mathrm{ml}^{-1}$ ) and 2 (MIC from 0.70 to $1.40 \mu \mathrm{g} \mathrm{ml}^{-1}$ ), unlike vancomycin (MIC from 2.97 to $760.68 \mu \mathrm{g} \mathrm{ml}^{-1}$ ), retained their antimicrobial activity against strains of VISA and VRSA strains. Furthermore, both thiazole compounds were more potent than teicoplanin against two VISA strains $\left(\mathrm{MIC}_{\text {Teicoplanin }}\right.$ from 0.94 to $7.52 \mu \mathrm{g} \mathrm{ml}^{-1}$ ) and all three VRSA strains tested $\left(\mathrm{MIC}_{\text {Teicoplanin }}\right.$ from 60.51 to $120.30 \mu \mathrm{g} \mathrm{ml}^{-1}$ ). Thus, 1 and 2 exhibits a selective advantage
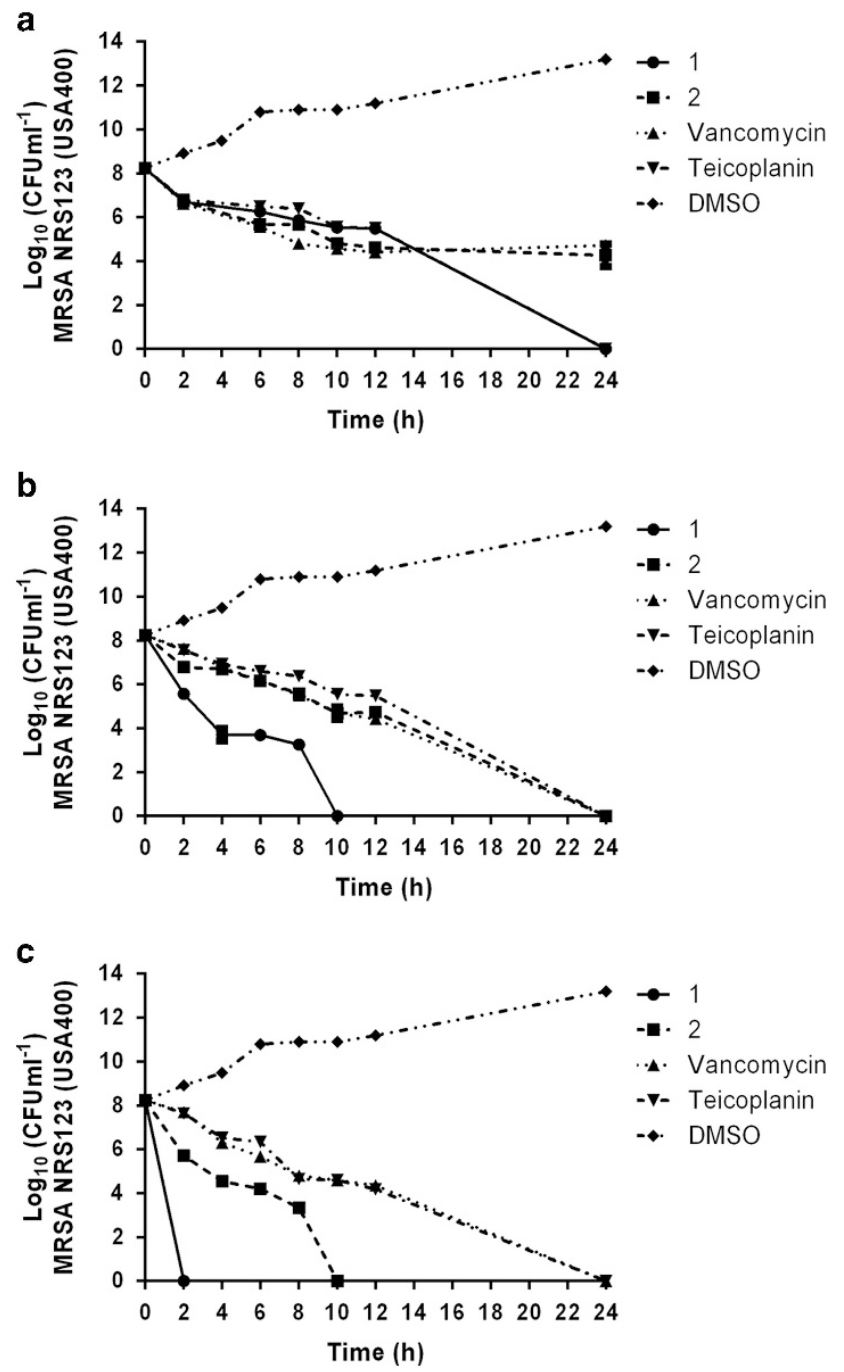

Figure 2 Time-kill analysis of the lead compound $\mathbf{1}$, derivative $\mathbf{2}$, teicoplanin, and vancomycin against methicillin-resistant Staphylococcus aureus (MRSA) strain NRS123 (USA400) at (a) $2 \times \mathrm{MIC}$, (b) $4 \times \mathrm{MIC}$ and (c) $8 \times$ MIC. Error bars represent s.d. values. over vancomycin and teicoplanin in their antibacterial activity against both VISA and VRSA.

Antimicrobial agents that exhibit bactericidal activity are hypothesized to contribute to a more rapid recovery from infection and a better clinical outcome, compared with their bacteriostatic counterparts. ${ }^{27}$ To ascertain whether the thiazole compounds were bacteriostatic or bactericidal, the MBC was determined. The MBC was calculated as the lowest concentration of compound/drug that produced $\mathrm{a} \geqslant 99.9 \%$ reduction in the bacterial cell count as compared with the initial inoculum. ${ }^{28}$ As Table 1 demonstrates, both thiazole compounds are bactericidal. Against five MRSA strains (NRS107, NRS119, NRS123, NRS385 and ATCC 43300), all VISA strains, and two VRSA strains (VRS1 and VRS4), 1 and 2 possess MBC values equivalent to their MICs or onefold higher than the MICs. This is similar to what is observed with vancomycin, a known bactericidal antibiotic, with MBC values equal to or onefold higher than the MICs for all MRSA and VISA strains tested. Teicoplanin exhibits MBC values equivalent to its MIC against two MRSA strains, fourfold higher than its MIC against two additional MRSA strains, and MBC values 16-fold higher than the MIC values against three strains of MRSA (NRS194, USA300 and USA400).

\section{Time-kill analysis of thiazole compounds and glycopeptide antibiotics}

In order to confirm that $\mathbf{1}$ and $\mathbf{2}$ were bactericidal agents, we next examined how rapidly the thiazole compounds were able to kill a high inoculum of MRSA. Using a standard time-kill assay, MRSA USA400 (NRS123), a predominant strain linked to many community-acquired MRSA infections, ${ }^{29}$ was exposed to 2,4 and $8 \times$ MIC of $\mathbf{1}, 2$, teicoplanin or vancomycin. Samples were collected at specific time points and transferred to TSA plates to determine the number of viable bacteria remaining posttreatment.

As depicted in Figure 2, both $\mathbf{1}$ and $\mathbf{2}$ exhibit bactericidal activity at all concentrations tested; however, the time to achieve a $3-\log _{10}$ reduction in $\mathrm{CFU} \mathrm{ml}{ }^{-1}$ differs depending on the concentration of the test agent. For compound 1, MRSA is completely eliminated after $24 \mathrm{~h}$ at $2 \times \mathrm{MIC}, 10 \mathrm{~h}$ at $4 \times \mathrm{MIC}$ and after only $2 \mathrm{~h}$ at $8 \times \mathrm{MIC}$. The analog 2 produces a $3-\log _{10}$ reduction in $\mathrm{CFU} \mathrm{ml}{ }^{-1}$ after $10 \mathrm{~h}$ at $2 \times$ MIC; however, it is not able to completely eradicate MRSA similar to the parent compound. At higher concentrations, 2 successfully eliminates MRSA completely after $24 \mathrm{~h}$ (at $4 \times \mathrm{MIC}$ ); at the highest concentration tested $(8 \times \mathrm{MIC})$, analog 2 proves superior to both antibiotics tested as it rapidly eliminates MRSA within $10 \mathrm{~h}$. Vancomycin required $24 \mathrm{~h}$ to completely eradicate MRSA at both 4 and $8 \times \mathrm{MIC}$; at $2 \times \mathrm{MIC}$, vancomycin produced a $3-\log _{10}$ reduction in $\mathrm{CFU} \mathrm{ml}{ }^{-1}$ within $24 \mathrm{~h}$ but was not able to eliminate all bacteria completely (similar to analog 2). These results are similar to what has been previously published regarding vancomycin's slow bactericidal activity. ${ }^{30}$ Teicoplanin required $24 \mathrm{~h}$ to completely eliminate MRSA at all three concentrations tested. Thus, in addition to retaining antimicrobial activity against VISA and VRSA strains, $\mathbf{1}$ and $\mathbf{2}$ possess an additional advantage over vancomycin and teicoplanin in their ability to rapidly kill MRSA, particularly at higher concentrations. Rapid bactericidal activity is an important factor in reducing the emergence of bacterial resistance to an antimicrobial agent, and is important clinically in preventing an infection from spreading. ${ }^{27}$ In addition, bactericidal agents have been shown both clinically and in in vivo studies to be superior to bacteriostatic agents for the treatment of certain invasive diseases, such as endocarditis. ${ }^{31}$ Furthermore, rapid bactericidal activity is an important quality for consideration in using a particular agent in combination with other antibiotics, such as 
vancomycin. ${ }^{30}$ The results from the time-kill assay provided valuable insight into the possibility that the thiazole compounds could be potentially paired with other antibiotics against MRSA, given $\mathbf{1}$ and $\mathbf{2}$ possess rapid bactericidal activity.

\section{Assessment of single-step resistance}

After confirming that compounds $\mathbf{1}$ and $\mathbf{2}$ possessed rapid bactericidal activity against MRSA, we next turned our attention to assessing the likelihood of MRSA would develop resistance quickly to these thiazole compounds. A single-step resistance selection experiment was performed by subculturing a high inoculum of MRSA $\left(>1 \times 10^{9} \mathrm{CFU} \mathrm{ml}{ }^{-1}\right)$ onto TSA plates containing $\mathbf{1}, 2$, vancomycin or teicoplanin at a concentration equivalent to $4 \times$ MIC. The likelihood of bacterial resistance arising (via spontaneous mutations in the bacterial genome) to these compounds/antibiotics was examined using five MRSA strains. Table 2 presents the mutation frequencies generated against each tested agent: for $1,1.19 \times 10^{-8}$ to $>1.73 \times 10^{-10}$; for $2,>1.73 \times 10^{-10}$ to $>2.33 \times 10^{-10}$; for teicoplanin, $2.73 \times 10^{-7}$ to $3.03 \times 10^{-9}$; and for vancomycin, $3.03 \times 10^{-10}$ to $>8.47 \times 10^{-10}$. The values obtained for teicoplanin and vancomycin are similar to what has been reported elsewhere. ${ }^{32}$

The thiazole compounds produce a similar mutation frequency as both teicoplanin and vancomycin. Interestingly, 2 demonstrates a mutation frequency similar to or better than vancomycin against the five MRSA strains tested. Even at lower $(2 \times$ MIC) concentrations, resistant mutants are difficult to isolate against this particular compound (data not published). It took 30 years to isolate a strain of $S$. aureus exhibiting resistance to vancomycin. ${ }^{1}$ Thus the results presented here support the notion that MRSA is unlikely to develop rapid resistance to the thiazole compounds, in particular compound 2. The data obtained from both the time-kill and single-step resistance selection experiments demonstrate that the thiazole compounds possess two important characteristics necessary for an ideal antibiotic for MRSA, rapid bactericidal activity and low potential for bacterial resistance development. ${ }^{33}$

\section{Combination testing of thiazole compounds with glycopeptide antibiotics}

Glycopeptide antibiotics, chiefly vancomycin, have been a principle source of treatment of MRSA infections for many years. ${ }^{33}$ However, extensive use of these antibiotics opens the door for the emergence of strains with reduced susceptibility to these antibiotics. ${ }^{30}$ The combination therapy, pairing vancomycin with another antimicrobial, has been used in the healthcare setting both to reduce the likelihood of resistant

Table 2 Single-step frequency of resistance determination for compounds 1 and 2, teicoplanin and vancomycin against methicillin-resistant $S$. aureus

\begin{tabular}{lcccc}
\hline & \multicolumn{5}{c}{ Compound/antibiotic name } \\
\cline { 2 - 5 } MRSA Strain & 1 & 2 & Teicoplanin & Vancomycin \\
\hline NRS107 & $>1.73 \times 10^{-10}$ & $>1.73 \times 10^{-10}$ & $1.91 \times 10^{-8}$ & $>1.73 \times 10^{-10}$ \\
NRS119 & $1.99 \times 10^{-8}$ & $>8.47 \times 10^{-10}$ & $2.73 \times 10^{-7}$ & $>8.47 \times 10^{-10}$ \\
NRS123 & $5.93 \times 10^{-9}$ & $>2.33 \times 10^{-10}$ & $2.21 \times 10^{-9}$ & $>2.33 \times 10^{-10}$ \\
(USA400) & & & & \\
NRS384 & $1.35 \times 10^{-8}$ & $>3.03 \times 10^{-10}$ & $3.03 \times 10^{-9}$ & $3.03 \times 10^{-10}$ \\
(USA300) & & & & \\
NRS385 & $1.19 \times 10^{-8}$ & $>3.31 \times 10^{-10}$ & $1.79 \times 10^{-8}$ & $>3.31 \times 10^{-10}$ \\
(USA500) & & & & \\
\hline
\end{tabular}

strains to vancomycin from rapidly emerging and to improve the morbidity associated with MRSA infections. For example, vancomycin has been combined with subinhibitory concentrations of clindamycin and linezolid to reduce toxins generated by $S$. aureus during infection. ${ }^{34,35}$ Identifying other antimicrobial partners capable of being paired with vancomycin can potentially prolong the clinical utility of this antibiotic.

To ascertain whether $\mathbf{1}$ and $\mathbf{2}$ have potential to be combined with vancomycin against MRSA, the checkerboard assay was used. ${ }^{22}$ In this assay, one antibiotic/compound is serially diluted along the abscissa followed by diluting the second antibiotic/compound along the ordinate in a 96-well plate. The FIC is then calculated as a ratio of the MIC of each antibiotic/compound when given in combination relative to the MIC of each antibiotic/compound given alone. The $\Sigma$ FIC is a summation of the FICs for each antibiotic/compound tested in combination. The $\Sigma$ FIC $\leqslant 0.50$ is indicative of synergism between the antibiotic and compound. Results from the checkerboard assay experiment are presented in Table 3. Both thiazole compounds were found to exhibit a synergistic relationship with vancomycin against six of the seven MRSA strains tested with $\Sigma$ FIC values ranging from 0.07 to 0.50 for 1 and 0.13 to 0.50 for 2 . At $1 / 4 \times$ MIC for 2 , a 16 -fold reduction in the MIC for vancomycin (when combined with 2 ) was observed for all six MRSA strains where synergy was detected (data not presented). As vancomycin is known to be a nephrotoxic agent, using a lower concentration of this drug in MRSA infections is highly desirable, as it has the potential benefit of reducing this side effect in patients. ${ }^{33}$ When tested against VISA, 1 failed to exhibit synergy with vancomycin, whereas 2 demonstrated a synergistic relationship with vancomycin against one strain (NRS19).

We were curious to explore whether the synergistic relationship observed was limited just to vancomycin or could be observed with other glycopeptide antibiotics as well. Teicoplanin was used to further explore the partnership between thiazole compounds and glycopeptide antibiotics. Interestingly, the checkerboard assay revealed that neither 1 nor 2 exhibited a synergistic relationship with teicoplanin against MRSA. This suggests that combination therapy involving the thiazole compounds may be limited to only being paired with vancomycin though further studies with other glycopeptide antibiotics are needed to confirm this observation. In addition, as vancomycin targets cell wall biosynthesis in S. aureus, it would be worthwhile to explore if a synergistic relationship would be observed between these thiazole compounds and other cell wall biosynthesis inhibitors (such as $\beta$-lactam antibiotics). Collectively, the results shed valuable insight into thiazole compounds serving as potential future partners with vancomycin against MRSA. This discovery can potentially prolong the usage of vancomycin as a therapeutic agent for MRSA infections by reducing the likelihood of strains developing resistance to vancomycin used in monotherapy.

\section{Resensitization of VRSA to glycopeptide antibiotics}

The emergence of $S$. aureus strains resistant to vancomycin presents an additional challenge to clinical care providers dealing with the growing epidemic of multidrug-resistant bacterial infections. Identifying clever strategies to prolong the use of current antibiotics against multidrugresistant bacteria is necessary. One strategy that has been explored recently is suppressing antibiotic resistance by resensitizing resistant bacteria using a secondary compound. ${ }^{23}$ As the thiazole compounds were found to possess a synergistic relationship with vancomycin against MRSA, we postulated that the thiazole compounds may be capable of resensitizing VRSA strains to vancomycin. Initially, the MIC of 1 and 2 was determined using the broth microdilution assay. Next, 
Table 3 The fractional inhibitory concentration index range of thiazole compounds 1 and 2 in combination with teicoplanin and vancomycin against methicillin-resistant and vancomycin-intermediate $S$. aureus

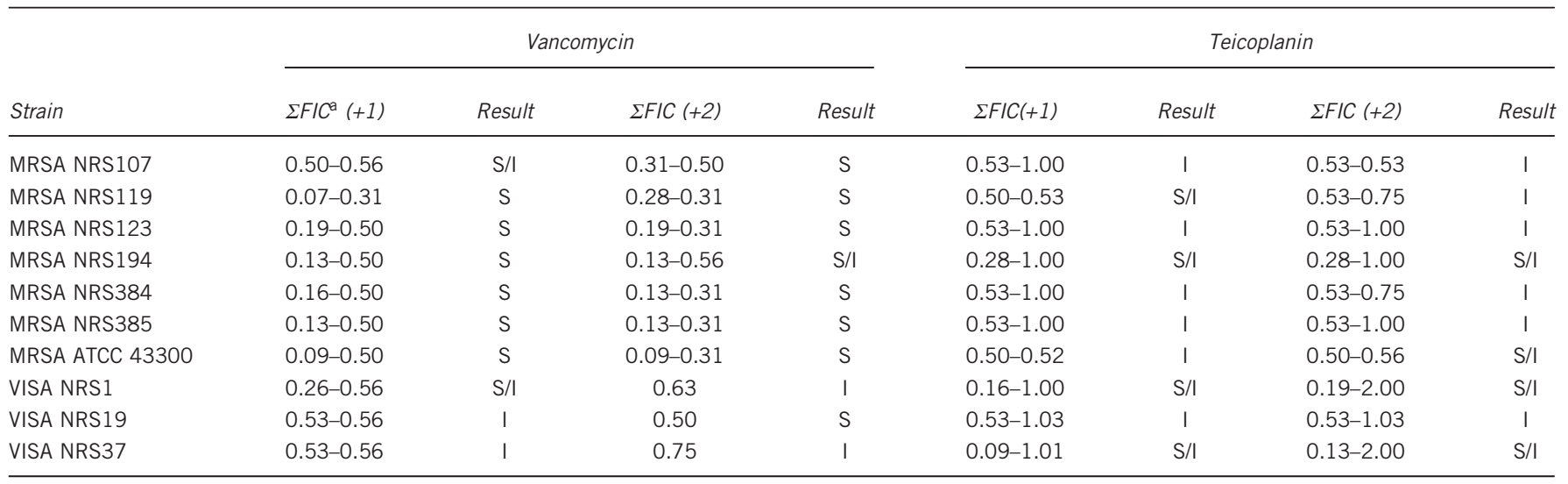

Abbreviations: A, antagonistic; $\Sigma$ FIC, fractional inhibitory concentration index; I, indifference; MRSA, methicillin-resistant $S$. aureus; S, synergistic; VISA, vancomycin-intermediate S. aureus. aResults for the FIC index are as follows: $\leqslant 0.5 \mathrm{~S} ;>0.5$ to $\leqslant 4.0 \mathrm{I} ;>4 \mathrm{~A}$. Results are reported from two independent experiments.

Table 4 Resensitization of vancomycin-resistant $S$. aureus (VRSA) to vancomycin and teicoplanin using a subinhibitory concentration $(1 / 2 \times$ MIC) of compound 1 or 2

\begin{tabular}{|c|c|c|c|c|c|c|c|c|}
\hline \multirow[b]{2}{*}{ Strain } & \multicolumn{2}{|c|}{ 1+Vancomycin } & \multicolumn{2}{|c|}{$2+$ Vancomycin } & \multicolumn{2}{|c|}{ 1+Teicoplanin } & \multicolumn{2}{|c|}{ 2+Teicoplanin } \\
\hline & Resensitization & $\Sigma F I C^{a}$ & Resensitization & $\Sigma F I C$ & Resensitization & $\Sigma F I C$ & Resensitization & $\Sigma F / C$ \\
\hline VRS1 & $<4$-fold & $>1.50$ & $<4$-fold & 1.00 & 0-fold & $>2.00$ & 64-fold & 0.63 \\
\hline VRS4 & $<4$-fold & 1.13 & 512-fold & 0.50 & 0-fold & 2.00 & 32-fold & 0.50 \\
\hline VRS5 & 4-fold & 1.25 & 512-fold & 0.50 & 2-fold & 1.50 & 32-fold & 0.50 \\
\hline
\end{tabular}

Abbreviation: $\Sigma F I C$, fractional inhibitory concentration index

a Results for the $\Sigma$ FIC are as follows: $\leqslant 0.5$ synergistic; $>0.5$ to $\leqslant 4.0$ indifference; $>4$ antagonistic.

MHB was inoculated with either compound 1 or 2 (at $1 / 2 \times$ MIC). Vancomycin was then serially diluted in both the inoculated media alone and media supplemented with the thiazole compounds. The MICs of vancomycin in the presence of the thiazole compounds was compared with vancomycin used alone. A fold reduction was calculated by dividing the MIC of vancomycin alone by the MIC of vancomycin + the thiazole compound.

As Table 4 presents, both thiazole compounds were capable of resensitizing VRSA to vancomycin. Compound 1 was able to produce a fourfold reduction in the MIC of vancomycin when the two agents were combined against VRSA. Amazingly, compound 2 proved to be superior to 1 , as it produced a 512-fold reduction in the MIC of vancomycin against two VRSA strains tested. Furthermore, compound 2 produced a 32 -fold reduction in the MIC of teicoplanin against two VRSA strains (VRS4 and VRS5) and a 64-fold reduction against strain VRS1. Thus, compound 2 was capable of resensitizing VRSA to both vancomycin and teicoplanin. Substitution of the alkane side chain (in 1) with a phenyl group (in 2) produced a marked improvement in the thiazole compounds' ability to resensitize VRSA to the effect of glycopeptide antibiotics. Using the checkerboard assay, we found that compound 2 exhibited a synergistic relationship with both vancomycin and teicoplanin against two VRSA strains (VRS4 and VRS5) with $\Sigma F I C=0.50$. Thus, compound 2 holds promise for future use to suppress vancomycin resistance in VRSA strains, prolonging the utility of glycopeptide antibiotics against these strains.

\section{S. epidermidis biofilm mass reduction}

Bacterial biofilms that form on the surface of indwelling medical devices, such as intravascular catheters, are a major problem in hospitals. These biofilms can lead to life-threatening bloodstream infections associated with high mortality and treatment costs. ${ }^{36}$ Staphylococci, primarily S. epidermidis and $S$. aureus, are responsible for many invasive infections which develop from bacterial biofilms that form on the surface of medical devices. ${ }^{3,37}$ Further exacerbating this problem, traditional antibiotics are not effective at disrupting these biofilms as cells present within the biofilm exhibit increased resistance to antibiotics. ${ }^{5}$ Identifying antimicrobials capable of disrupting these biofilms is necessary to combat this growing problem.

Recent studies have demonstrated that thiazole and thiazolidinone compounds possess the ability to disrupt bacterial biofilms. ${ }^{38,39}$ To examine whether the potential therapeutic application of $\mathbf{1}$ and $\mathbf{2}$ could be expanded beyond just inhibition of planktonic bacteria, the ability of both thiazole compounds to disrupt staphylococcal biofilm was analyzed. First, to confirm the thiazole compounds were capable of inhibiting planktonic bacteria, the MIC of each compound and vancomycin against a biofilm-forming clinical isolate of methicillinresistant $S$. epidermidis was assessed using the broth microdilution technique. Compounds 1 and $\mathbf{2}$ were found to inhibit bacterial growth at 2.77 and $0.61 \mu \mathrm{g} \mathrm{ml}^{-1}$, respectively (Table 1). Vancomycin inhibited growth of planktonic $S$. epidermidis at a concentration of $0.74 \mu \mathrm{g} \mathrm{ml}^{-1}$. Next, to determine whether compounds $\mathbf{1}$ and $\mathbf{2}$ had the potential to disrupt staphylococcal biofilm, the crystal violet reporter assay was used against a mature $S$. epidermidis biofilm. ${ }^{24}$ As Figure 3 demonstrates, 1 (at $8 \times \mathrm{MIC}$ ) and 2 (at $32 \times \mathrm{MIC}$ ) significantly disrupted S. epidermidis biofilm, reducing the biofilm mass by $56.7 \%$ and $65.2 \%$, respectively. These compounds proved to be far superior to vancomycin; even at $128 \times \mathrm{MIC}$, vancomycin was only able to reduce S. epidermidis biofilm mass by $21.5 \%$. The thiazole compounds thus 


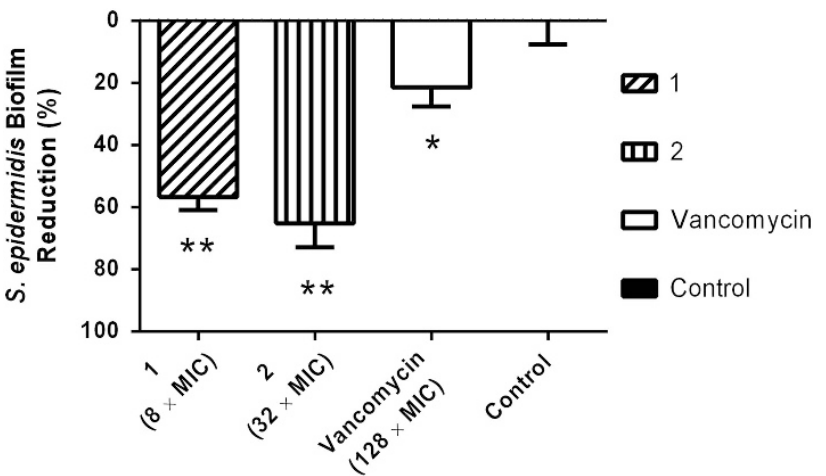

Figure 3 Efficacy of thiazole compounds 1 and 2 and vancomycin (all at $64 \mu \mathrm{m}$ ) in disrupting an established methicillin-resistant S. epidermidis ATCC 35984 biofilm. Bacteria were incubated at $37^{\circ} \mathrm{C}$ in Mueller-Hinton broth (MHB) medium supplemented with glucose for $24 \mathrm{~h}$ to allow biofilm formation. Wells were subsequently rinsed with phosphate-buffered saline before MHB containing different concentrations of each test agent was added. Following incubation for $24 \mathrm{~h}$, wells were washed again and left to dry. The adherent biofilm was stained with crystal violet and then the dye was extracted with ethanol before turbidity was measured at $595 \mathrm{~nm}$. Data are presented as percentage of biofilm mass reduction compared with untreated wells (control). All experiments were done in triplicate. ${ }^{*}$ Indicates data are statistically different when compared with the control $(P<0.05)$. $* *$ Indicates the data are statistically different from the vancomycin-treated wells $(P<0.05)$

possess anti-biofilm activity and are capable of disrupting adherent staphylococci biofilm much better than a traditional antibiotic, vancomycin.

Pharmacokinetic analysis of compound 2

Assessment of a compound's drug-like properties is important early in drug development to identify and address potential issues, especially those associated with aqueous solubility and permeability. Previously we reported the lead thiazole compound $\mathbf{1}$ possessed moderate aqueous solubility $\left(21.6 \mu \mathrm{g} \mathrm{ml}^{-1}\right)$ but poor permeability across a biological membrane (Caco-2 apparent permeability, $P_{\text {app }}$ $\left.(\mathrm{A} \rightarrow \mathrm{B})=0.0 \times 10^{-6} \mathrm{~cm} \mathrm{~s}^{-1}\right) .{ }^{16}$ We were interested to examine whether compound 2 , containing a phenyl ring substitution in place of the linear alkane side chain present in $\mathbf{1}$, would exhibit an improved pharmacokinetic profile. Initially, a turbidometric solubility screen was used to assess the maximum concentration compound 2 that was able to dissolve in an aqueous buffer (PBS). Table 5 demonstrates that the phenyl ring substitution resulted in a significant decrease in the aqueous solubility of compound $2\left(2.70 \mu \mathrm{g} \mathrm{ml}^{-1}\right)$ relative to 1 . After determining that compound 2 exhibited poor aqueous solubility, this compound's ability to passively diffuse across a biological membrane was assessed.

The Caco- 2 permeability assay was used to determine whether compound 2 was more permeable than compound 1 . Compound 2 $(10 \mu \mathrm{M})$ was added to the apical (A) surface of a Caco-2 bilayer and the rate of transfer of the compound to the basolateral (B) surface was measured. The process was then repeated in reverse to assess the rate of transfer from the B to A direction. As Table 6 demonstrates, the rate of transfer of compound 2 from the apical to basolateral surface was not measurable $\left(P_{\text {app }}(\mathrm{A} \rightarrow \mathrm{B})=0.0 \times 10^{-6} \mathrm{~cm} \mathrm{~s}^{-1}\right)$. However, the rate of transfer from the $\mathrm{B}$ to $\mathrm{A}$ surface was measured to be $1.2 \times 10^{-6}$ $\mathrm{cm} \mathrm{sec}^{-1}$. This is similar to what is observed with the poorly permeable drug control ranitidine $\left(P_{\text {app }}(\mathrm{B} \rightarrow \mathrm{A})=1.7 \times 10^{-6} \mathrm{~cm} \mathrm{~s}^{-1}\right)$. Thus, the results indicate that compound 2 does not exhibit improved
Table 5 Evaluation of solubility of thiazole compound 2, reserpine, tamoxifen, and verapamil in phosphate-buffered saline (PBS)

\begin{tabular}{lc}
\hline Compound tested & Solubility limit $\left(\mu g \mathrm{ml}^{-1}\right)^{\mathrm{a}}$ \\
\hline $\mathbf{2}$ & 2.70 \\
Reserpine & 19.05 \\
Tamoxifen & 5.80 \\
Verapamil & $>227.30$ \\
\hline aSolubility limit corresponds to the highest concentration of test compound where no precipitate \\
was detected.
\end{tabular}

Table 6 Evaluation of physicochemical properties (apparent permeability) of thiazole compound 2 , ranitidine, warfarin, and talinolol via the Caco-2 permeability assay

\begin{tabular}{lccc}
\hline $\begin{array}{l}\text { Compound } \\
\text { tested }\end{array}$ & $\begin{array}{c}\text { Mean } A \rightarrow B^{\mathrm{a}} \mathrm{P}_{\text {app }} \\
\left(10^{-6} \mathrm{~cm} \mathrm{~s}^{-1}\right)\end{array}$ & $\begin{array}{c}\text { Mean } B \rightarrow A^{\mathrm{b}} P_{a p p} \\
\left(10^{-6} \mathrm{~cm} \mathrm{~s}^{-1}\right)\end{array}$ & $\begin{array}{l}\text { Efflux } \\
\text { ratio }\end{array}$ \\
\hline 2 & $0.0^{\mathrm{d}}$ & 1.2 & $>2$ \\
Ranitidine & 0.2 & 1.7 & 8.5 \\
Warfarin & 27.6 & 11.1 & 0.4 \\
Talinolol & 0.1 & 8.3 & 83
\end{tabular}

a Mean $\mathrm{A} \rightarrow \mathrm{B} P_{\mathrm{app}}=$ mean apparent permeability of test compound from apical to basolateral surface.

${ }^{\mathrm{b}}$ Mean $\mathrm{B} \rightarrow \mathrm{A} P_{\mathrm{app}}=$ mean apparent permeability of test compound from basolateral to apical surface.

${ }^{C}$ Effluxratio $=\frac{P a p p(B \rightarrow A)}{P(B P}(A) B$

${ }^{d}$ Compound not detected in receiver compartment (peak below limit of detection); permeability may be underestimated.

permeability relative to 1 . The discrepancy between the rate of transfer of compound 2 across the basolateral and apical surfaces results in an efflux ratio $>2$; this suggests that 2 may be a substrate for an efflux transporter (such as P-glycoprotein). One method to overcome the effect of efflux transporters is to saturate the transporters, by using a higher concentration than $10 \mu \mathrm{M}$ of compound 2 used for the assay, thus permitting passive transfer of the compound across the apical surface of the membrane.

Although limited solubility and permeability characteristics are not encouraging to consider biologically active compounds as drug candidates for subsequent clinical steps, recent formulation technology has been able to overcome such limitations to propel valuable compounds with similar kinetic profiles into the market. For instance, the orally administrated protease inhibitor telaprivir possesses an aqueous solubility profile similar to compound 2 . By using a spray drying dispersion technique, telaprivir's water solubility, permeability and the consequent bioavailability were markedly improved. ${ }^{40}$ Moreover, formulators have more techniques to handle poor water solubility, such as using the solvent/antisolvent method. ${ }^{41}$ By shedding light on the limited pharmacokinetic profile of compound 2, we are opening a gate for formulators to investigate their time and effort improving the pharmacokinetic profile of this very promising antimicrobial agent.

\section{CONCLUSION}

We have successfully developed an approach to synthesize phenylthiazole compounds with potent antibacterial activity against MRSA, VISA and VRSA. The most potent derivative 2 exhibited MIC values ranging from 0.70 to $1.40 \mu \mathrm{g} \mathrm{ml}^{-1}$ and $\mathrm{MBC}$ values ranging from 1.40 to $11.17 \mu \mathrm{g} \mathrm{ml}^{-1}$ against MRSA, VISA and VRSA. Both compounds 1 and 2 rapidly eliminated MRSA within $10 \mathrm{~h}$ at $8 \times \mathrm{MIC}$, whereas vancomycin required $24 \mathrm{~h}$; in addition, both thiazole compounds 
exhibited low resistance frequencies, similar to vancomycin. Lead 1 behaved synergistically when combined with vancomycin exhibiting $\Sigma$ FIC ranging from 0.07 to 0.50 against six MRSA strains, whereas derivative 2 behaved synergistically with vancomycin exhibiting $\Sigma$ FIC ranging from 0.09 to 0.50 against six MRSA strains. Interestingly, compound 2 demonstrated the ability to resensitize two VRSA strains to vancomycin and teicoplanin reducing their MIC by 512 -fold and 32-fold, respectively. In addition, both compounds 1 and 2 exhibited strong anti-biofilm activity reducing adherent $S$. epidermidis biofilm by $56.7 \%$ and $65.2 \%$, respectively. As compound 2 did not demonstrate good solubility or permeability properties, incorporating advanced formulation techniques are a must to improve its pharmacokinetic profile. In addition, further derivatives will be constructed with the aim of improving the thiazole compounds' drug-like properties while maintaining their strong antibacterial properties. Collectively, the thiazole compounds prepared here have the versatility to potentially be used for multiple therapeutic applications including being used alone or in combination with vancomycin against multidrug-resistant staphylococci, to resensitize VRSA to vancomycin or to disrupt mature staphylococcal biofilms.

\section{ACKNOWLEDGEMENTS}

We thank the Network of Antimicrobial Resistance in Staphylococcus aureus program supported under NIAID/NIH Contract \# HHSN272200700055C for providing MRSA, VISA and VRSA strains used in this study.

1 CDC. Antibiotic Resistance Threats in the United States, 2013, 1-114 (Centers for Disease Control and Prevention, Atlanta, GA, USA, 2013).

2 Moran, G. J. et al. Methicillin-resistant S. aureus infections among patients in the emergency department. New Engl. J. Med. 355, 666-674 (2006).

3 Wang, R. et al. Staphylococcus epidermidis surfactant peptides promote biofilm maturation and dissemination of biofilm-associated infection in mice. J. Clin. Invest. 121, 238-248 (2011)

4 Davies D. Understanding biofilm resistance to antibacterial agents. Nat. Rev. Drug Discov. 2003; 2: 114-122.

5 Mah, T. F. \& O'Toole, G. A. Mechanisms of biofilm resistance to antimicrobial agents Trends Microbiol. 9, 34-39 (2001).

6 Chambers, H. F. Community-associated MRSA-resistance and virulence converge. New Engl. J. Med. 352, 1485-1487 (2005)

7 Moran, G. J., Amii, R. N., Abrahamian, F. M. \& Talan, D. A. Methicillin-resistant Staphylococcus aureus in community-acquired skin infections. Emerg. Infect. Dis. 11, 928-930 (2005)

8 Hiramatsu, K. Vancomycin-resistant Staphylococcus aureus: a new model of antibiotic resistance. Lancet Infect. Dis. 1, 147-155 (2001).

9 Hays, S. J. et al. Substituted 2-benzothiazolamine as sodium flux inhibitorsquantitative structure-activity-relationships and anticonvulsant activity. J. Pharm. Sci. 83, 1425-1432 (1994).

10 Das, J. et al. Discovery of 2-amino-heteroaryl-benzothiazole-6-anilides as potent p56 (Ick) inhibitors. Bioorg. Med. Chem. Lett. 13, 2587-2590 (2003).

11 Hutchinson, I., Bradshaw, T. D., Matthews, C. S., Stevens, M. F. \& Westwell, A. D. Antitumour benzothiazoles. Part 20: 3'-cyano and 3'-alkynyl-substituted 2-(4'-aminophenyl)benzothiazoles as new potent and selective analogues. Bioorg. Med. Chem. Lett. 13, 471-474 (2003)

12 Paget, C. J., Kisner, K., Stone, R. L. \& DeLong, D. C. Heterocyclic substituted ureas II. Immunosuppressive and antiviral activity of benzothiazole- and benzoxazoleureas. J. Med Chem. 12, 1016-1018 (1969).

13 Darwish, E. S., Fattah, A. M. A., Attaby, F. A. \& Al-Shayea, O. N. Synthesis and antimicrobial evaluation of some novel thiazole, pyridone, pyrazole, chromene, hydrazone derivatives bearing a biologically active sulfonamide moiety. Int. J. Mol. Sci. 15, 1237-1254 (2014).
14 Desai, N. C., Bhatt, N., Somani, H. \& Trivedi, A. Synthesis, antimicrobial and cytotoxic activities of some novel thiazole clubbed 1,3,4-oxadiazoles. Eur. J. Med. Chem. 67 54-59 (2013).

15 Sadek, B., Al-Tabakha, M. M. \& Fahelelbom, K. M. S. Antimicrobial prospect of newly synthesized 1,3-thiazole derivatives. Molecules 16, 9386-9396 (2011).

16 Mohammad, H. et al. Discovery and characterization of potent thiazoles versus methicillin- and vancomycin-resistant staphylococcus aureus. J. Med. Chem. 57, 1609-1615 (2014).

17 Mayhoub, A. S. et al. An investigation of phenylthiazole antiflaviviral agents. Bioorg. Med. Chem. 19, 3845-3854 (2011).

18 Clinical and Laboratory Standards Institute. Methods for Dilution Antimicrobial Susceptibility Tests for Bacteria That Grow Aerobically - Seventh Edition: Approved Standard M7-A7 (Wayne, PA, USA, 2011).

19 Messick, C. R., Rodvold, K. A. \& Pendland, S. L. Modified time-kill assay against multidrug-resistant Enterococcus faecium with novel antimicrobial combinations. J. Antimicrob. Chemoth. 44, 831-834 (1999).

20 Baldoni, D., Haschke, M., Rajacic, Z., Zimmerli, W. \& Trampuz, A. Linezolid alone or combined with rifampin against methicillin-resistant Staphylococcus aureus in experimental foreign-body infection. Antimicrob. Agents. Chemother. 53 1142-1148 (2009).

21 Nagai, K. et al. In vitro selection of resistance to clinafloxacin, ciprofloxacin, and trovafloxacin in Streptococcus pneumoniae. Antimicrob. Agents. Chemother. 44, 2740-2746 (2000).

22 Orhan, G., Bayram, A., Zer, Y. \& Balci, I. Synergy tests by E test and checkerboard methods of antimicrobial combinations against Brucella melitensis. J. Clin. Microbiol. 43, 140-143 (2005).

23 Furlani, R. E., Yeagley, A. A. \& Melander, C. A flexible approach to 1,4-disubstituted 2-aminoimidazoles that inhibit and disperse biofilms and potentiate the effects of B-lactams against multi-drug resistant bacteria. Eur. J. Med. Chem. 2013; 62: 59-70. 24 O'Toole, G. A. Microtiter dish biofilm formation assay. J. Vis. Exp. 2011; 2437.

25 Mohamed, M. F., Hamed, M. I., Panitch, A. \& Seleem, M. N. Targeting methicillinresistant staphylococcus aureus with short salt-resistant synthetic peptides. Antimicrob. Agents Chemother. 58, 4113-4122 (2014).

26 NCCLS. Methods for Dilution Antimicrobial Susceptibility Tests for Bacteria that Grow Aerobically. NCCLS Approved Standard M7-A9 (NCCLS: Wayne, PA, USA, 2012).

27 Alder, J. \& Eisenstein, B. The advantage of bactericidal drugs in the treatment of infection. Curr. Infect. Dis. Rep. 6, 251-253 (2004).

28 Pankey, G. A. \& Sabath, L. D. Clinical relevance of bacteriostatic versus bactericidal mechanisms of action in the treatment of Gram-positive bacterial infections. Clin. Infect. Dis. 38, 864-870 (2004)

29 McDougal, L. K. et al. Pulsed-field gel electrophoresis typing of oxacillin-resistant Staphylococcus aureus isolates from the United States: establishing a national database. J. Clin. Microbiol. 41, 5113-5120 (2003).

30 Deresinski, S. Vancomycin in combination with other antibiotics for the treatment of serious methicillin-resistant Staphylococcus aureus infections. Clin. Infect. Dis. 49, 1072-1079 (2009).

31 Finberg, R. W. et al. The importance of bactericidal drugs: future directions in infectious disease. Clin. Infect. Dis. 39, 1314-1320 (2004).

32 Kosowska-Shick, K. et al. Activity of telavancin against staphylococci and enterococci determined by MIC and resistance selection studies. Antimicrob. Agents Chemother. 53, 4217-4224 (2009).

33 Nguyen, H. M. \& Graber, C. J. Limitations of antibiotic options for invasive infections caused by methicillin-resistant Staphylococcus aureus: is combination therapy the answer? J. Antimicrob. Chemoth. 65, 24-36 (2010).

34 Dumitrescu, 0. et al. Effect of antibiotics, alone and in combination, on PantonValentine leukocidin production by a Staphylococcus aureus reference strain. Clin. Microbiol. Infect. 14, 384-388 (2008).

35 Stevens, D. L. et al. Impact of antibiotics on expression of virulence-associated exotoxin genes in methicillin-sensitive and methicillin-resistant Staphylococcus aureus. J. Infect. Dis. 195, 202-211 (2007).

36 Leonidou, L. \& Gogos, C. A. Catheter-related bloodstream infections: catheter manage ment according to pathogen. Int. J. Antimicrob. Agents 36, S26-S32 (2010).

37 Vuong, C. \& Otto, M. Staphylococcus epidermidis infections. Microbes Infect. 4, 481-489 (2002).

38 More, P. G., Karale, N. N., Lawand, A. S., Narang, N. \& Patil, R. H. Synthesis and antibiofilm activity of thiazole Schiff bases. Med. Chem. Res. 23, 790-799 (2014).

39 Rane, R. A., Sahu, N. U. \& Shah, C. P. Synthesis and antibiofilm activity of marine natural product-based 4-thiazolidinones derivatives. Bioorg. Med. Chem. Lett. 22 7131-7134 (2012)

40 Kwong, A. D., Kauffman, R. S., Hurter, P. \& Mueller, P. Discovery and development of telaprevir: an NS3-4A protease inhibitor for treating genotype 1 chronic hepatitis C virus. Nat. Biotechnol. 29, 993-1003 (2011).

41 Allegrini P. \& Brunoldi E. Process for the preparation of a viral protease inhibitor in amorphous form. Google Patents W02013153055 A1 (2013). 